\title{
Estrutura diamétrica e espacial de espécies madeireiras de importância econômica na Amazônia
}

\author{
Diametric and spatial structure of wood species of economic importance in \\ the Amazon
}

\author{
Diego dos Santos Vieira (D), Karla Mayara Almada Gomes² (D), Lizandra Elizeário dos Santos² (D), \\ Marcio Leles Romarco de Oliveira ${ }^{1}$ (1) João Ricardo Vasconcellos Gama² (1), \\ Evandro Luiz Machado Mendonça ${ }^{1}$ (D), Bruno Oliveira Lafetá ${ }^{3}$ (D), Cristiane Coelho de Moura ${ }^{1}$ (D), \\ Axa Emanuelle Simões Figueiredo ${ }^{4}$ (D) \\ ${ }^{1}$ Universidade Federal dos Vales do Jequitinhonha e Mucuri - UFVJM, Diamantina, MG, Brasil \\ ${ }^{2}$ Universidade Federal do Oeste do Pará - UFOPA, Santarém, PA, Brasil \\ ${ }^{3}$ Instituto Federal de Educação, Ciência e Tecnologia de Minas Gerais - IFMG, São João Evangelista, MG, Brasil \\ ${ }^{3}$ Instituto Nacional de Pesquisa da Amazônia - INPA, Manaus, AM, Brasil
}

Como citar: Vieira, D. S., Gomes, K. M. A., Santos, L. E., Oliveira, M. L. R., Gama, J. R. V., Mendonça, E. L. M., Lafetá, B. O., Moura, C. C., \& Figueiredo, A. E. S. (2021). Estrutura diamétrica e espacial de espécies madeireiras de importância econômica na Amazônia. Scientia Forestalis, 49(129), e3438.

https://doi.org/10.18671/scifor.v49n129.21

\begin{abstract}
Resumo
O objetivo desse estudo foi avaliar a estrutura diamétrica e o padrão espacial de quatro espécies madeireiras de importância econômica na Amazônia. $O$ estudo foi realizado em nove Unidades de Trabalho de 100 ha cada, inseridas na Unidade de Produção Anual $n^{\circ} 8$ da Unidade de Manejo Florestal Samambaia, pertencente à Cooperativa Mista da Floresta Nacional do Tapajós, município de Belterra. Os dados foram coletados por meio de um inventário $100 \%$, com mapeamento, em coordenadas cartesianas, de todas as espécies com diâmetro a 1,30 m do solo igual ou superior a $20 \mathrm{~cm}$. A estrutura diamétrica das espécies foi obtida por meio de análises de agrupamento e discriminante. A partir dessas estruturas foram testadas cinco funções de densidade probabilística (Weibull-3P, Gamma, Normal, Lognormal e Exponencial). O ajuste foi avaliado por meio do teste de Kolmogorov-Smirnov, a 95\% de probabilidade e histogramas. O padrão espacial foi obtido por meio da função K de Ripley. As espécies apresentaram distribuição diamétrica tendendo à normalidade, o que sugere problemas de regeneração ocasionados, provavelmente, pela baixa produção de sementes, condições edafoclimáticas desfavoráveis ou redução da fauna polinizadora. A espécie Hymenaea courbaril apresentou padrão espacial aleatório, enquanto Manilkara huberi e Handroanthus serratifolius exibiram padrões agregados. A espécie Mezilaurus itauba apresentou padrão variando em função da distância, isto é, aleatório para distâncias inferiores a $\pm 100 \mathrm{~m}$, agregado até aproximadamente $825 \mathrm{~m}$ e aleatório até $1.500 \mathrm{~m}$. Os padrões registrados são, provavelmente, resultados das síndromes de dispersão das espécies e condições edafoclimáticas da UPA.
\end{abstract}

Palavras-chave: Função K de Ripley; Handroanthus serratifolius; Mezilaurus itauba; Hymenaea courbaril.

\footnotetext{
Abstract
}

The objective of this study was to evaluate the diametric structure and spatial pattern of four timber species of economic importance in the Amazon. The study was carried out in nine Work Units of 100 ha each, inserted in the Annual Production Unit $n^{\circ} 8$ of the Forest Management Unit Samambaia, belonging to the Mixed Cooperative of the Tapajós National Forest, Belterra municipality. Data were collected through a $100 \%$ inventory, with Cartesian coordinates mapping of all species with a diameter of $1.30 \mathrm{~m}$

Fonte de financiamento: Nenhuma.

Conflito de interesse: Nada a declarar.

Autor correspondente: diegovieir4@gmail.com

Recebido: 4 agosto 2019.

Aceito: 2 abril 2020.

Editor: Paulo Henrique Müller Silva. 
equal to or greater than $20 \mathrm{~cm}$. Diametric structure of the species was obtained through cluster and discriminant analyzes. From these structures five functions of probability density (Weibull-3P, Gamma, Normal, Lognormal and Exponential) were tested. The fit was assessed using the Kolmogorov-Smirnov test, 95\% probability and histograms. Spatial pattern was obtained through Ripley's K-function. The species showed a diametric distribution tending to normal, which suggests regeneration problems, probably caused by low seed production, unfavorable edapho-climatic conditions or reduction of pollinating fauna. Hymenaea courbaril presented a random spatial pattern, while Manilkara huberi and Handroanthus serratifolius exhibited aggregate patterns. Mezilaurus itauba presented a pattern varying in relation to distance, that is, random for distances less than $\pm 100 \mathrm{~m}$, aggregated up to approximately $825 \mathrm{~m}$ and random up to 1,500 m. Probably species dispersal syndromes and soil and climatic conditions of PAU affected the results reported in this study.

Keywords: Ripley's K function; Handroanthus serratifolius; Mezilaurus itauba; Hymenaea courbaril.

\section{INTRODUÇÃO}

O estímulo à produção sustentável de produtos madeireiros tornou-se uma importante ferramenta para vencer o desafio de conciliar desenvolvimento econômico com manutenção de recursos naturais numa perspectiva socialmente justa (Schwartz et al., 2008). Todavia, fazse necessário a realização de estudos ecológicos que forneçam bases reais para o ótimo manejo das espécies comerciais da Amazônia. Outra importante razão para sua realização é a exploração extrativista ilegal (Ferraz et al., 2002), que atualmente é promissora e praticamente inevitável. Logo, existe a necessidade de conservação dos recursos naturais madeireiros, e para tanto faz-se necessário a intensificação de estudos sobre a ecologia das espécies do bioma Amazônia, principalmente, para o desenvolvimento de técnicas silviculturais adequadas, conservando não só as árvores, mas os animais que se alimentam de suas sementes (Abreu et al., 2014).

Os estudos ecológicos envolvem, dentre outros aspectos, a avaliação da estrutura diamétrica e o padrão espacial, além de servirem de subsídio para inferências sobre o passado e o futuro de espécies arbóreas (Abreu et al., 2014). A estrutura diamétrica fornece informações sobre o estoque de madeira disponível para colheita (Cysneiros et al., 2017), auxilia na tomada de decisões sobre a necessidade de reposição florestal (Abreu et al., 2014) e permite a avaliação da regeneração de espécies comerciais e não comerciais. Ela permite, ainda, realizar inferências sobre o ingresso, mortalidade e o histórico de desenvolvimento de espécies arbóreas (Gül et al., 2005; Alves Júnior et al., 2007), definir critérios de exploração, tais como sistema e intensidade de exploração (Orellana et al., 2014), além de criar estratégias de manutenção, recuperação e conservação, de maneira que a floresta não sofra grandes distúrbios ecológicos (Costa et al., 2019).

As estruturas diamétricas são descritas por uma série de funções de densidade probabilística (FDPs), que permitem obter a probabilidade de as árvores ocorrerem dentro de um determinado intervalo de diâmetro (Cysneiros et al., 2017; Dalla Lana et al., 2013). É importante lembrar ainda que, devido às características de cada espécie, há necessidade de que as FDPs utilizadas sejam devidamente testadas e selecionadas (Téo et al., 2014). O padrão espacial, por sua vez, fornece indicações sobre os processos biológicos subjacentes e o estado funcional dos ecossistemas, refletindo parte de sua história e demostrando seus efeitos sobre as interações entre plantas e outros organismos (e.g. herbívoros) e entre outros organismos e seus predadores (Stamatellos \& Panourgias, 2005). O conhecimento do padrão espacial de espécies arbóreas favorece ainda a aplicação de práticas de conversação (Dantas et al., 2017) e manejo florestal, pois facilita atividades de inventário e planejamento da colheita, minimizando custos de locomoção de equipamentos e da frente de trabalho (Moraes et al., 2017).

Outra importante aplicação do padrão espacial é relacionada ao planejamento de inventários amostrais, pois a forma como a espécies distribuem-se na área é de fundamental importância para definição do sistema e desenho de amostral selecionado, por meio da adequação do tamanho e forma das unidades amostrais que terão grande influência sobre o valor do coeficiente de variação do inventário e, consequentemente, dos custos (Vieira et al., 
2017). O padrão espacial auxilia também na definição de árvores matrizes em sistemas de manejo baseados na regeneração natural (Martins et al., 2003). Todavia, é importante que o padrão espacial seja determinado por meio de metodologias eficientes, pois a maioria dos estudos fazem uso de índices baseados no uso de unidades amostrais (e.g. Payandeh e Morisita), que são fortemente influenciados pelo tamanho da parcela e intensidade de amostragem (Zhang et al., 2013), além de exibirem o comportamento de apenas parte da população, as parcelas. $O$ ideal é que se utilize técnicas que avaliem todas as árvores da área e que demonstre seu comportamento em função da distância, tais como as funções G e K de Ripley (Vieira et al., 2017).

As inferências obtidas a partir da distribuição diamétrica e espacial são, dessa forma, imprescindíveis para o bom manejo e conservação de espécies arbóreas, principalmente aquelas de valor comercial e altos níveis de exploração. As espécies Handroanthus serratifolius (Vahl) S.Grose (Ipê-amarelo), Manilkara huberi (Ducke) A.Chev (Maçaranduba), Hymenaea courbaril L. (Jatobá) e Mezilaurus itauba (Meisn.) Taub. ex Mez (Itaúba), por exemplo, são as mais exploradas nos estados da Amazônia e, por tanto, necessitam de estudos ecológicos que auxiliem seu manejo florestal, pois o alto preço de suas madeiras pode acarretar, a longo prazo, a redução de suas populações a níveis críticos. O maior exemplo do decréscimo de indivíduos é a $M$. itauba que, segundo a Lista de Espécies Brasileira Ameaçadas de Extinção e a Lista Vermelha da União Internacional para Conservação da Natureza, encontra-se classificada como uma espécie de risco de extinção de natureza alto, isto é, "vulnerável" (International Union for Conservation of Nature, 2018).

As demais, $H$. serratifolius, $M$. huberi e $H$. courbaril, embora sejam classificadas como de "menor preocupação" devem ser avaliadas, principalmente, para sua assegurar sua perpetuidade na estrutura da floresta, pois nem todos os mecanismos de sustentabilidade das florestas manejadas são adequadamente abordados pelos gestores e pela atual legislação florestal brasileira. A partir desse contexto, o objetivo desse estudo foi avaliar e determinar a estrutura diamétrica e o padrão espacial de quatro espécies de ampla comercialização, a saber: H. serratifolius, M. huberi, H. courbaril e M. itauba, a fim de auxiliar no manejo e conservação dessas espécies na Amazônia. A hipótese desse estudo é de que todas as espécies possuem estrutura diamétrica em forma exponencial negativo e padrão espacial agregado.

\section{MATERIAL E MÉTODOS}

\section{Área de estudo}

O estudo foi realizado em nove Unidades de Trabalho (UTs), de 100 ha cada, inseridas na Unidade de Produção Anual (UPA) $n^{\circ} 8$ da Unidade de Manejo Florestal Samambaia $\left(3^{\circ} 31^{\prime} 01^{\prime \prime} \mathrm{S} 55^{\circ} 04^{\prime} 23^{\prime \prime} \mathrm{O}\right)$, pertencente à Cooperativa Mista da Floresta Nacional do Tapajós (Coomflona), município de Belterra, oeste do estado do Pará, Brasil (BR). O clima da região, segundo a classificação de Köppen, é do tipo Ami, com temperatura e precipitação média anual de $25,5^{\circ} \mathrm{C}$ e $1.820 \mathrm{~mm}$, respectivamente (Alvares et al., 2013). O relevo é pouco acidentado e apresenta topografia variando de suavemente ondulada a ondulada, com predomínio do Latossolo Amarelo Distrófico (Santos et al., 2017). A vegetação é classificada como Floresta Ombrófila Densa de Terra Firme, caracterizando-se pela dominância de indivíduos arbóreos de grande porte e pela abundância de lianas lenhosas, palmeiras e epífitas.

\section{Coleta de dados}

A coleta de dados foi realizada por meio de um inventário 100\%, com mapeamento, em coordenadas cartesianas, de todas as espécies com diâmetro a 1,30 $\mathrm{m}$ do solo igual ou superior a $20 \mathrm{~cm}$ (DAP $\geq 20 \mathrm{~cm}$ ). O inventário incluiu diversas espécies comerciais e não comerciais, porém para essa pesquisa foram selecionadas apenas quatro espécies, a saber: 
Mezilaurus itauba (Meisn.) Taub. ex Mez (Itaúba), Handroanthus serratifolius (Vahl) S.Grose (Ipêamarelo), Manilkara huberi (Ducke) A.Chev (Maçaranduba) e Hymenaea courbaril L. (Jatobá), as quais foram selecionadas por suas importâncias econômicas, ecológicas e sociais no estado do Pará. A UPA foi dividida em nove Unidades de Trabalho (UTs), de 100 ha cada (1.000 x $1.000 \mathrm{~m})$, nas quais foram abertas picadas paralelas na direção $\mathrm{N}$-S, distantes $50 \mathrm{~m}$ entre elas (Vieira et al., 2017).

Nas picadas foram instaladas balizas a cada $25 \mathrm{~m}$, com a respectiva metragem em relação à origem, para posterior registro da coordenada $\mathrm{Y}$ das árvores. A coordenada $\mathrm{X}$ dos indivíduos arbóreos foi obtida por meio da distância entre a árvore e a respectiva linha da picada. Ao final de cada picada, movia-se para a seguinte e executava-se a mesma rotina de trabalho, e assim sucessivamente, até completar a última picada da UPA (Vieira et al., 2017). As árvores amostradas foram identificadas pelo nome vulgar e coletadas informações sobre o DAP e altura comercial $(\mathrm{Hc})$. O procedimento de identificação das espécies foi inicialmente realizado por mateiros experientes, utilizando-se de observações das folhas, casca, lenho e exsudações. A confirmação da identificação taxonômica foi realizada por meio especialistas da Universidade Federal do Oeste do Pará (UFOPA). O sistema de classificação adotado foi o APG IV (Angiosperm Phylogeny Group, 2016).

\section{Análise de dados}

\section{Estrutura diamétrica}

O procedimento de construção da estrutura diamétrica das espécies foi realizado por meio de técnicas multivariadas (Vieira et al., 2017). Logo, as árvores foram organizadas em ordem crescente de diâmetro, em classes com amplitude de $1 \mathrm{~cm}$. Em seguida, elaborou-se uma matriz $\mathrm{D}$ de dados de diâmetro, em que cada variável representava o diâmetro da i-ésima árvore na j-ésima classe de diâmetro. A matriz $\mathrm{D}$ foi o input para as análises de agrupamento e discriminante (Souza et al., 2003; Souza \& Souza, 2004). Os critérios de agrupamento adotados para definir os grupos constituídos por classes de $1 \mathrm{~cm}$ de diâmetro, denominadas classes diamétricas, foram a distância euclidiana e o método de Ward (Souza et al., 2014; Souza \& Souza, 2004). A escolha do método multivariado foi pautada na sua maior acurácia para avaliar a distribuição diamétrica de espécies, tendo como objetivo conhecer sua autoecologia, pois as amplitudes das classes diamétricas possuem fundamentos estatísticos válidos.

A partir do dendrograma resultante da análise de agrupamento foi traçada uma linha de corte, de modo a formar quinze classes diamétricas. A análise discriminante de Fisher e a estatística Wilk's Lambda foram utilizadas, posteriormente, para confirmação da classificação ou reclassificação dos agrupamentos formados na análise de agrupamento (Souza et al., 2014). Foram testadas ainda cinco funções de densidade probabilística (FDPs) (Weibull-3P, Gamma, Normal, Lognormal e Exponencial) para obtenção das distribuições de frequência de árvores em cada classe de diâmetro. Os parâmetros da função Weibull-3P foram obtidos pelo método da máxima verossimilhança, enquanto as demais foram ajustadas pelo método dos momentos. $\mathrm{O}$ ajuste foi avaliado por meio do teste de aderência de Kolmogorov-Smirnov (K-S), a 95\% de probabilidade e histogramas. Os dois melhores ajustes foram representados juntos aos histogramas de frequência observada.

\section{Função K de Ripley}

O padrão espacial foi obtido por meio da Função K(s) de Ripley. A função K(s) de Ripley foi obtida por meio de um círculo com raio $s$ de $5 \mathrm{~m}$ centrado em cada árvore, em que o número de vizinhos presentes na área desse círculo foi contado. Variando o raio $s$ a uma distância máxima de $1.500 \mathrm{~m}$, detectou-se o padrão espacial da espécie em diferentes escalas de distância (Vieira et al., 2017). Casos particulares ocorreram quando as árvores estavam próximas às bordas da UPA, uma vez que, por ser a função K(s) acumulativa e computar todas 
as distâncias entre todos os eventos, as árvores próximas à borda de raio $s$ maior que o limite do mapa não poderiam ser interpretadas como se não houvesse vizinhos. Os vizinhos existiam, mas por estarem fora dos limites da área não foram computados. Consequentemente, o número de árvores vizinhas a árvores próximo aos limites do mapa seria mais baixo do que para as demais, ocasionando um viés no cálculo do estimador da função K(s) de Ripley. À vista disso, utilizou-se o estimador da função K com correção isotrópica de bordadura (Ripley, 1977).

$\mathrm{K}(\mathrm{s})=\frac{1}{\hat{\lambda}_{\mathrm{n}}} \sum_{\mathrm{i}=\mathrm{i}=1}^{\mathrm{n}} \sum_{\mathrm{i}}^{\mathrm{n}} \frac{1}{\mathrm{~W}_{\mathrm{I}}}\left(\mathrm{x}_{\mathrm{i}}, \mathrm{x}_{\mathrm{j}}\right) \mathrm{I}\left(\left\|\mathrm{x}_{\mathrm{i}}-\mathrm{x}_{\mathrm{j}}\right\|<\mathrm{s}\right)$, para $\mathrm{i} \neq \mathrm{j}$ e $\mathrm{S}>0$

em que: $n$ é o número de árvores na região de estudo; $X_{i}$ e $X_{j}$ são as coordenadas dos pontos do mapa; $\left\|\mathrm{X}_{\mathrm{i}}-\mathrm{X}_{\mathrm{j}}\right\|$ é a distância euclidiana entre a localização $\mathrm{X}_{i}$ e $\mathrm{X}_{j}$; s é um vetor arbitrário de distância; $W_{l} I\left(X_{i}, X_{j}\right)$ é a função de correção para efeito de borda, que representa a proporção da circunferência com centro em $\mathrm{X}_{\mathrm{i}}$ e com raio $\left\|\mathrm{X}_{\mathrm{i}}-\mathrm{X}_{\mathrm{j}}\right\|$ que está fora da região de estudo; $\hat{\lambda}=\mathrm{n} / \mathrm{A} \mid$ é o número de árvores dividido pela área da região de estudos, sendo um estimador não viciado da intensidade do processo; e I(U) é uma função indicadora que assume valor 1 sempre que a condição $U$ for verdadeira e zero quando for falsa.

Objetivando-se analisar os dados graficamente, foram construídos envelopes de confiança por meio de 1.000 simulações Monte Carlo, realizadas com o modelo de completa aleatoriedade espacial (CAE). Posteriormente, foi calculada a função $\mathrm{K}(\mathrm{s})$ para os resultados das simulações, armazenando-se os valores mínimos e máximos da estimativa de $\mathrm{K}(\mathrm{s})$, utilizados para gerar intervalos de confiança a $99 \%$ de probabilidade. Para facilitar a análise, os valores da função $\mathrm{K}(\mathrm{s})$ foram transformados para $\mathrm{L}(\mathrm{s})$, de acordo com a formulação apresentada a seguir, e distribuídos em um gráfico, em que os eixos das abscissas e ordenadas representam, respectivamente, as distâncias $s$ acumuladas e os valores transformados da função K (Ripley, 1979).

$\mathrm{L}(\mathrm{s})=\sqrt{\frac{\mathrm{K}(\mathrm{s})}{\pi}-\mathrm{s}}$

Como resultado, obteve-se envelopes de confiança identificado por duas linhas limites pontilhadas, uma positiva e outra negativa. Se os valores observados de L(s) estiverem dentro dos envelopes construídos, o padrão espacial é aleatório, caso contrário rejeita-se a hipótese nula e assume-se que o padrão espacial dos indivíduos é agregado, quando passar do limite superior do envelope, e regular, quando passar do limite inferior (Ripley, 1979). Os cálculos foram realizados com o auxílio do software R versão 3.3.2 (R Core Team, 2018), sendo as análises de agrupamento e discriminante obtidas a partir do pacote vegan (Oksanen et al., 2018) e a função K de Ripley por meio do pacote splancs (Rowlingson \& Diggle, 2018).

\section{RESULTADOS E DISCUSSÃO}

\section{Estrutura diamétrica}

O inventário registrou a densidade de $0,33,0,34,0,66$ e 0,50 ind ha-1 de $M$. itauba, H. serratifolius, M. huberi, $H$. courbaril, respectivamente. Os valores observados na UPA são inconsistentes com os registrados em diferentes áreas da Amazônia, o que pode ser efeito das condições edafoclimáticas e, até mesmo, níveis antrópicos a que estão submetidas, que são determinantes para o desenvolvimento dessas espécies. Os resultados de Sontag (2017), em quatro áreas ( $A, B, C$ e D) ao longo da Amazônia, para árvores com DAP $\geq 40 \mathrm{~cm}$, demonstraram que a espécie $M$. huberi comumente possui alta e baixa densidade, 
dependendo da região de ocorrência, e que é mais sensível às condições climáticas que $H$. serratifolius e $H$. courbaril. Ainda segundo a autora, a espécie $H$. serratifolius rotineiramente apresenta densidade semelhante independentemente da região, o que não foi notado na UPA, que apresentou valor superior aos registrados nas áreas $A\left(0,03\right.$ ind ha-1), B $\left(0,05\right.$ ind ha $\left.^{-1}\right)$, $C(0,02$ ind ha-1 $)$ e $D\left(0,08\right.$ ind $\left.h^{-1}\right)$, mesmo avaliando apenas as árvores de DAP $\geq 40 \mathrm{~cm}$. Aquelas com DAP $<40 \mathrm{~cm}$ somaram apenas $4,7 \%$ de toda a densidade da $H$. serratifolius na UPA.

A densidade registrada para $M$. huberi foi menor que as obtidas por Sontag (2017) nas áreas $\mathrm{A}\left(0,54\right.$ ind $\left.\mathrm{ha}^{-1}\right)$ e $\mathrm{B}\left(3,94\right.$ ind ha $\left.^{-1}\right)$ e maior que as das áreas $\mathrm{C}\left(0,02\right.$ ind ha- $\left.{ }^{-1}\right)$ e $\mathrm{D}(0,04$ ind $\left.\mathrm{ha}^{-1}\right)$, confirmando a existência de uma variação do número de árvores por hectare independentemente do diâmetro mínimo de inclusão ou região amostrada, pois a densidade da área $B$, que avaliou apenas árvores com $D A P \geq 40 \mathrm{~cm}$, foi superior à registrada na UPA, que avaliou indivíduos com DAP $\geq 20 \mathrm{~cm}$. Outras regiões do estado do Pará registraram densidades de 1,20, (Pinheiro et al., 2007) e 0,58 ind ha-1 (Almeida et al., 2001). A $\pm 4.000 \mathrm{~m}$ de distância da UPA foram registradas 2,40 ind ha-1 (DAP $\geq 20 \mathrm{~cm}$ ) (Schwartz et al., 2014). A densidade de $H$. courbaril foi maior que aquelas registradas nas áreas $A\left(0,07\right.$ ind ha-1 $\left.{ }^{-1}\right), B$ $\left(0,37\right.$ ind ha- $\left.{ }^{-1}\right), C(0,09$ ind ha-1 $)$ e $D$ (nenhuma ind ha- ${ }^{-1}$ (Sontag, 2017) e muito próximo aos 0,59 e 0,40 ind ha-1 obtidos no nordeste (Pinheiro et al., 2007) e oeste do estado do Pará (Schwartz et al., 2014).

O número de árvores por hectare de $M$. itauba foi menor que as 1,50 e 1,23 ind ha ${ }^{-1}$ registradas nas proximidades de SINOP (Braz et al., 2018; Ebert et al., 2016), mas próxima à 0,40 ind ha-1 obtida por Schwartz et al. (2014), a \pm 8.000 m de distância da UPA. Ainda que as densidades de $M$. itauba, $H$. serratifolius, $M$. huberi, $H$. courbaril sejam menores que as registradas em outras regiões da Amazônia, seus valores admitem sua exploração, pois há mais de 3 árvores com DAP $\geq 50 \mathrm{~cm}$ a cada 100 ha para cada espécie na UPA, exigência mínima determinada pelos órgãos ambientais para que a espécie não seja considerada "rara" e seja explorável (Brasil, 2006). Todavia, somente o critério de raridade não determina a capacidade de exploração de uma espécie. É importante que se avalie a estrutura diamétrica e o padrão espacial, que fornecem informações sobre a condição das árvores nas classes diamétricas abaixo do diâmetro mínimo de corte, indicando se haverá árvores para reposição daquelas exploradas, assegurando sua regeneração em fluxo contínuo (Cysneiros et al., 2017; Orellana et al., 2014).

A metodologia adotada para construção da estrutura diamétrica foi eficaz. As análises de agrupamento mostraram que, avaliando os dendrogramas com linha de corte em níveis de 50 a 100\%, foram registradas, em todas as espécies, 15 grupos homogêneos e distintos, denominados de classes diamétricas, as quais apresentaram diferentes amplitudes. Observou-se também que o arranjo das árvores individuais em amplitude de 1,0 cm permitiu agrupar, sequencialmente, em ordem crescente as classes diamétricas. As análises discriminantes de Fisher entre as classes diamétricas demostraram diferenças significativas para todas as espécies, com valores da estatística Wilk's Lambda próximos a zero, evidenciando que $100 \%$ das classes diamétricas para as espécies $M$. itauba, H. serratifolius e M. huberi foram corretamente classificadas. A espécie $H$. courbaril, apresentou $99,4 \%$ dos indivíduos classificados de forma correta nas classes diamétricas. As classes erroneamente classificadas foram realocadas, a partir de suas probabilidades posteriores, e novamente realizadas as análises discriminantes de Fisher, para obter 100\% das classes corretamente classificadas.

As espécies apresentaram distribuição diamétrica tendendo à normalidade, isto é, baixo número de árvores nas classes diamétricas menores e elevada densidade nas classes intermediárias, com redução acentuada no sentido das maiores classes diamétricas (Figura 1), o que indica descontinuidade de regeneração das espécies (Orellana et al., 2014). Ademais, do ponto de vista estatístico, essa tendência foi descrita por várias FDPs. As estruturas das espécies $M$. itauba, H. serratifolius e $H$. courbaril, por exemplo, foram descritas pelas funções Gamma, Lognormal, Normal e Weibull-3P, enquanto a espécie M. huberi foi descrita pelas funções Gamma, Lognormal e Weibull-3P (Tabela 1). Apesar de duas ou mais funções 
descrevem o comportamento da maioria das espécies observou-se que a estrutura diamétrica de $M$. itauba, $H$. serratifolius, $M$. huberi e $H$. courbaril foi melhor representada pelas funções Weibull-3P, Gamma, Lognormal e Weibull-3P, respectivamente. A acurácia dessas FDPs é amplamente citada na literatura (Cysneiros et al., 2017; Dalla Lana et al., 2013; Orellana et al., 2014; Téo et al., 2014; Wendling et al., 2011), devido à flexibilidade de descrever curvas de distribuição de diferentes formas e conformações. A função Exponencial não se mostrou adequada para descrever a estrutura diamétrica de nenhuma das espécies avaliadas $\left(D_{C A L}>\right.$ $D_{T A B}$ ), o que permite afirmar que o comportamento exponencial negativo (J-invertido) não é característico dessas populações.

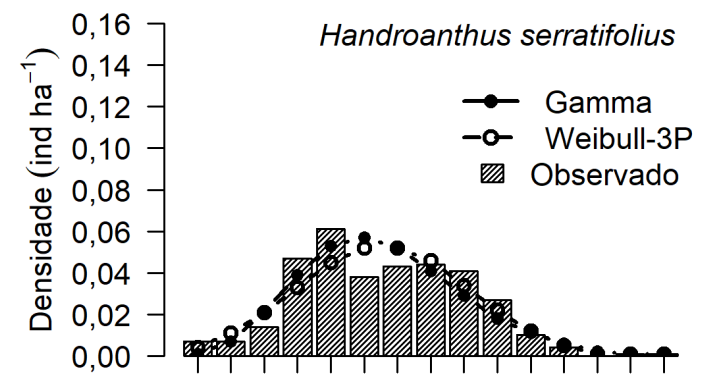

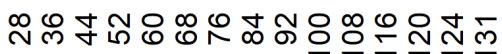

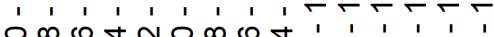

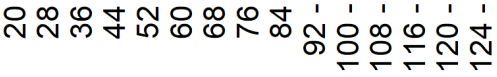

Classes diamétricas $(\mathrm{cm})$

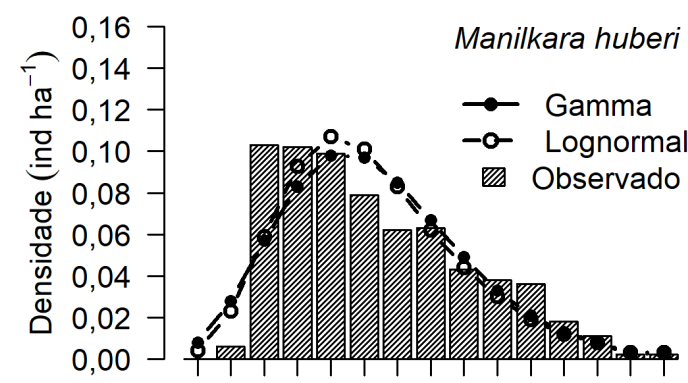

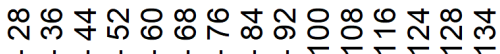

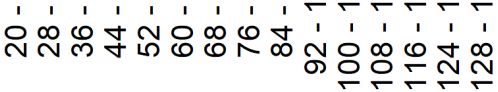

Classes diamétricas $(\mathrm{cm})$

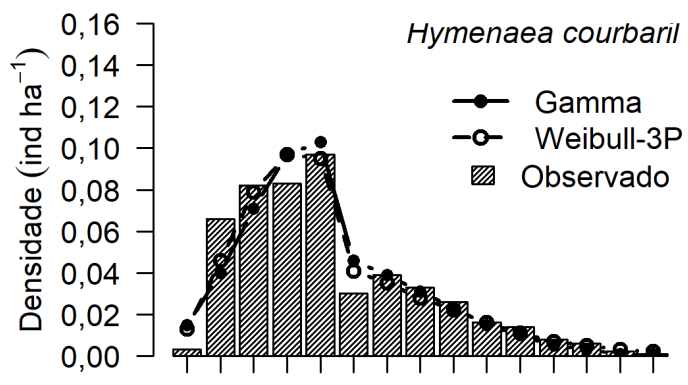

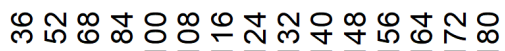
ín

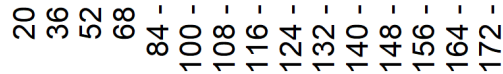

Classes diamétricas $(\mathrm{cm})$

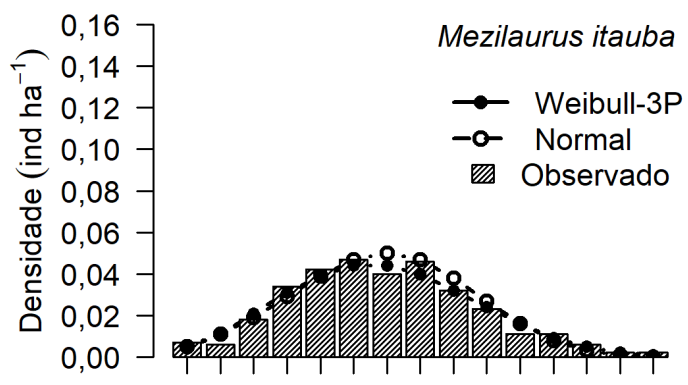

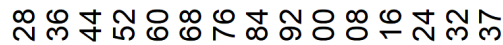

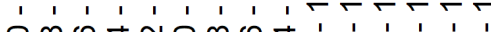

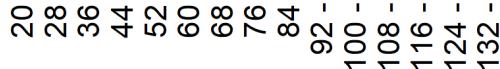

Classes diamétricas $(\mathrm{cm})$

Figura 1. Estrutura diamétricas observadas e estimadas pelas funções de densidade probabilística de melhor desempenho das quatro espécies.

A distribuição tendendo à normalidade é consequência do baixo recrutamento de plantas jovens, ocasionado, possivelmente, pela baixa produção de sementes e taxa de germinação, além da mudança do estágio sucessional da floresta de inicial para mais avançado. Ainda pode ser resultado do ritmo lento de desenvolvimento das árvores, o que é associado à espécies de ciclo longo com pequeno número de sementes e germinação lenta, e das alterações climáticas, as quais tem gerado condições de menor precipitação, o que afeta a capacidade de alguma espécies de reproduzirem e estabelecerem árvores jovens (Vieira et al., 2018). Outra hipótese é relacionada à Síndrome da Floresta Vazia, que ocorre quando a fauna polinizadora e dispersora de sementes se encontra em declínio, afetando os processos de polinização e produção de sementes, bem como a dispersão eficiente de propágulos, causando restrições no recrutamento de novas árvores (Carvalho, 2010).

Avaliando a distribuição diamétrica de Carapa guianensis Aubl. e Bertholletia excelsa Bonpl. na mesma área, Vieira et al. $(2017,2018)$ também registraram tendências à normalidade e atribuíram esse comportamento às características heliófila, longeva e dominante das 
espécies, às elevadas taxas intra e interanual de mortalidade da espécie, que associada às fortes variações temporais na precipitação tem ocasionado oscilações na estrutura diamétrica à longo prazo, diminuindo o ingresso de árvores menores, e à ausência de perturbações naturais e antrópicas na área. Ainda segundo Vieira et al. (2018), as sementes de C. guianensis são facilmente predadas por insetos, principalmente do gênero Hypsipyla sp., cujas larvas atacam os frutos, fazendo galerias, danificando mais de 39\% da produção de sementes, o que reduz a taxa de germinação e, consequentemente, o estabelecimento das plântulas (Pinto et al., 2013).

Tabela 1. Abundâncias e ranking das funções de densidade probabilística ajustadas.

\begin{tabular}{|c|c|c|c|c|c|c|}
\hline \multirow{2}{*}{ Espécies } & \multirow{2}{*}{-} & \multicolumn{5}{|c|}{ Funções de densidade probabilística } \\
\hline & & Exponencial & Gamma & Lognormal & Normal & Weibull-3P \\
\hline \multirow{3}{*}{ M. itauba } & $\mathrm{DA}_{\mathrm{EST}}$ & 0,207 & 0,339 & 0,336 & 0,337 & 0,322 \\
\hline & $\mathrm{D}_{\mathrm{TAB}}$ & 0,0945 & 0,0945 & 0,0945 & 0,0945 & 0,0945 \\
\hline & $\mathrm{D}_{\mathrm{CAL}}$ & $0,2584^{*(5)}$ & $0,0373^{\text {ns }(3)}$ & $0,0573^{\text {ns (4) }}$ & $0,0317^{\text {ns }(2)}$ & $0,0177^{\text {ns }(1)}$ \\
\hline \multirow{3}{*}{ H. serratifolius } & $\mathrm{DA}_{\mathrm{EST}}$ & 0,205 & 0,340 & 0,337 & 0,339 & 0,340 \\
\hline & $\mathrm{D}_{\mathrm{TAB}}$ & 0,0918 & 0,0918 & 0,0918 & 0,0918 & 0,0918 \\
\hline & $\mathrm{D}_{\mathrm{CAL}}$ & $0,2878 *(5)$ & $0,0518^{\text {ns }(1)}$ & $0,0627^{\text {ns (3) }}$ & $0,0677^{\text {ns }(4)}$ & $0,0549^{\text {ns (2) }}$ \\
\hline \multirow{3}{*}{ M. huberi } & $\mathrm{DA}_{\mathrm{EST}}$ & 0,399 & 0,651 & 0,649 & 0,644 & 0,625 \\
\hline & $\mathrm{D}_{\mathrm{TAB}}$ & 0,0662 & 0,0662 & 0,0662 & 0,0662 & 0,0662 \\
\hline & $\mathrm{D}_{\mathrm{CAL}}$ & $0,2522 *(5)$ & $0,0478^{\text {ns (2) }}$ & $0,0462^{\mathrm{ns}(1)}$ & $0,0959 *(4)$ & $0,0654^{\text {ns (3) }}$ \\
\hline \multirow{3}{*}{ H. courbaril } & $\mathrm{DA}_{\mathrm{EST}}$ & 0,335 & 0,497 & 0,492 & 0,493 & 0,499 \\
\hline & $\mathrm{D}_{\mathrm{TAB}}$ & 0,0760 & 0,0760 & 0,0760 & 0,0760 & 0,0760 \\
\hline & $\mathrm{D}_{\mathrm{CAL}}$ & $0,2273 *(5)$ & $0,0507^{\text {ns }(2)}$ & $0,0624^{\text {ns }(4)}$ & $0,0533^{\text {ns }(3)}$ & $0,0291^{\text {ns }(1)}$ \\
\hline
\end{tabular}

Legenda: $\mathrm{DA}_{\mathrm{EST}}=$ densidade absoluta estimada; $\mathrm{D}_{\mathrm{TAB}}$ e $\mathrm{D}_{\mathrm{CAL}}=\mathrm{D}$ tabelado e calculado, respectivamente, do teste Kolmogorov-Smirnov; ${ }^{\mathrm{ns}}=$ não significativo, a 95\% de probabilidade; * = significativo, a 95\% de probabilidade; (1), (2), .... $(n)=$ ranking das funções de densidade probabilística

Acredita-se ainda que se a mesma área e espécies fossem avaliadas, porém com DAP $\geq$ $10 \mathrm{~cm}$, que é o utilizado na maioria dos inventários realizados na Amazônia, a ausência de regenerantes continuaria, pois os novos indivíduos registrados não compensariam os baixos valores de densidade observados nas classes diamétricas iniciais das quatro espécies. Ademais, mesmo que a suposta classe diamétrica de $10-20 \mathrm{~cm}$ apresentasse maior densidade que as demais, só corroboraria a hipótese de que em algum período houve alterações locais (e.g. baixa produção de sementes, pragas, e condições edafoclimáticas desfavoráveis), que afetaram o ingresso de plântulas para classes de DAP maiores que $20 \mathrm{~cm}$. Outros estudos realizados na Amazônia também registraram estruturas diamétricas tendendo à normalidade para as espécies M. huberi (Hirai et al., 2008; Cunha et al., 2012), M. itauba (Braz et al., 2018), H. courbaril (Cunha et al., 2012) e H. serratifolius (Cunha et al., 2012), corroborando os resultados encontrados na UPA.

O baixo número de árvores nas classes menores característico de distribuições tendendo à normalidade é preocupante do ponto de vista ecológico, pois espécies tropicais de ciclo longo ou heliófilas, em florestas em estágio avançado de sucessão, habitualmente não apresentam árvores regenerantes nas classes diamétricas menores, indicando uma possível 
ausência da população conforme a evolução da floresta. Todavia, Schwartz et al. (2014), avaliando a influência da exploração de impacto reduzido (EIR) em áreas próximas da UPA, observou que as espécies $H$. courbaril, parcialmente tolerante à sombra, e M. huberi, totalmente tolerantes à sombra, tiveram aumentos na densidade de regenerantes após a EIR, o que é benéfico para a conservação dessas espécies a longo prazo, pois há maiores probabilidades de mudas ou plântulas dessas espécies alcançarem classes diamétricas maiores devido à maior incidência de luz (Oliveira et al., 2011). A considerar o caráter heliófilo, supõem-se que o mesmo ocorra para as espécies $H$. serratifolius e $M$. itauba. A exploração sustentável aliada a ações silviculturais seria, dessa forma, um dos meios para aumentar a regeneração natural e garantir a permanência dessas espécies na estrutura da floresta (Azevedo et al., 2008a).

\section{Padrão espacial}

O padrão espacial das espécies arbóreas tropicais é determinado pelos diversos fatores intrínsecos e extrínsecos à espécie (Lan et al., 2012). A dispersão de sementes e as condições edafoclimáticas são os mais importantes e explicam quase todos os padrões aleatórios e agregados encontrados na Amazônia (Abreu et al., 2014). O envelope de confiança obtido a partir da função $\mathrm{K}(\mathrm{s})$ demonstra que a espécie $H$. courbaril atende à hipótese de completa aleatoriedade espacial (CAE) e, portanto, é distribuída aleatoriamente na UPA (Figura 2), contrariando a expectativa de que espécies com síndrome de dispersão barocórica e zoocórica tendem à apresentarem-se distribuídas de forma agregada (Jorge et al., 2015), além dos resultados de outros estudos que registraram padrões agregados para árvores de H. courbaril com DAP $\geq 15 \mathrm{~cm}$ (Lima-Ribeiro et al., 2011), DAP $\geq 20 \mathrm{~cm}$ (Schwartz et al., 2014) e DAP $\geq 45 \mathrm{~cm}$ (Sontag, 2017). A diferença é provavelmente consequência do tamanho e localização da região inventariada, pois quanto maior a área amostrada maior o tamanho e o número de agregados identificados.

Avaliando o padrão espacial de árvores de $H$. courbaril (DAP $\geq 45 \mathrm{~cm}$ ) em duas áreas próximas na Amazônia, Sontag (2017) observou que o padrão espacial variou significativamente de uma região para outra, isto é, de altamente agregado para completamente inexistente (i.e. completa ausência de árvores de espécie). Ainda segundo a autora o resultado foi consequência do tamanho e posição de ambas as áreas dentro da Área de Manejo Florestal (AMF), pois uma das áreas ficou localizada na região com ausência de H. courbaril. Outra explicação, porém, menos provável, devido aos outros estudos citados anteriormente, é relacionada ao diâmetro mínimo do inventário, pois árvores de maior porte, que provavelmente tem maior idade e estão estabelecidas na floresta, ocorrem independentes umas das outras, ou seja, aleatoriamente.

A espécie $M$. itauba atende parcialmente à hipótese CAE e apresenta padrão espacial variando em função da distância máxima de $1.500 \mathrm{~m}$. Até $\pm 100 \mathrm{~m} \mathrm{M}$. itauba apresentou padrão aleatório, passando para um padrão espacial agregado até aproximadamente $825 \mathrm{~m}$, e a partir daí retornam um padrão aleatório até $1.500 \mathrm{~m}$ (Figura 2). As variações de padrão na escala de distância são geralmente relacionadas ao porte arbóreo das árvores, uma vez que árvores mais jovens tendem a ocorrer mais próximas umas das outras, formando agregados. À medida que elas crescem, ocorre aumento da competição e poucas alcançam os maiores diâmetros, ficando mais distanciadas umas das outras, o que leva a uma distribuição aleatória (Araújo et al., 2014). Ebert et al. (2016) encontraram resultados semelhantes para uma população de $M$. itauba no sul da Amazônia que apresentou padrão aleatório e agregado para maiores e menores distâncias, respectivamente, o que foi resultado do crescimento vegetativo, dispersão zoocórica e da baixa disponibilidade de nutrientes essenciais as árvores de $M$. itauba. A falta de nutrientes associada aos períodos de secas intensas registradas na Amazônia nos últimos anos também limita o ingresso de árvores nas classes diamétricas maiores, tornando-as aleatoriamente mais distribuídas. 

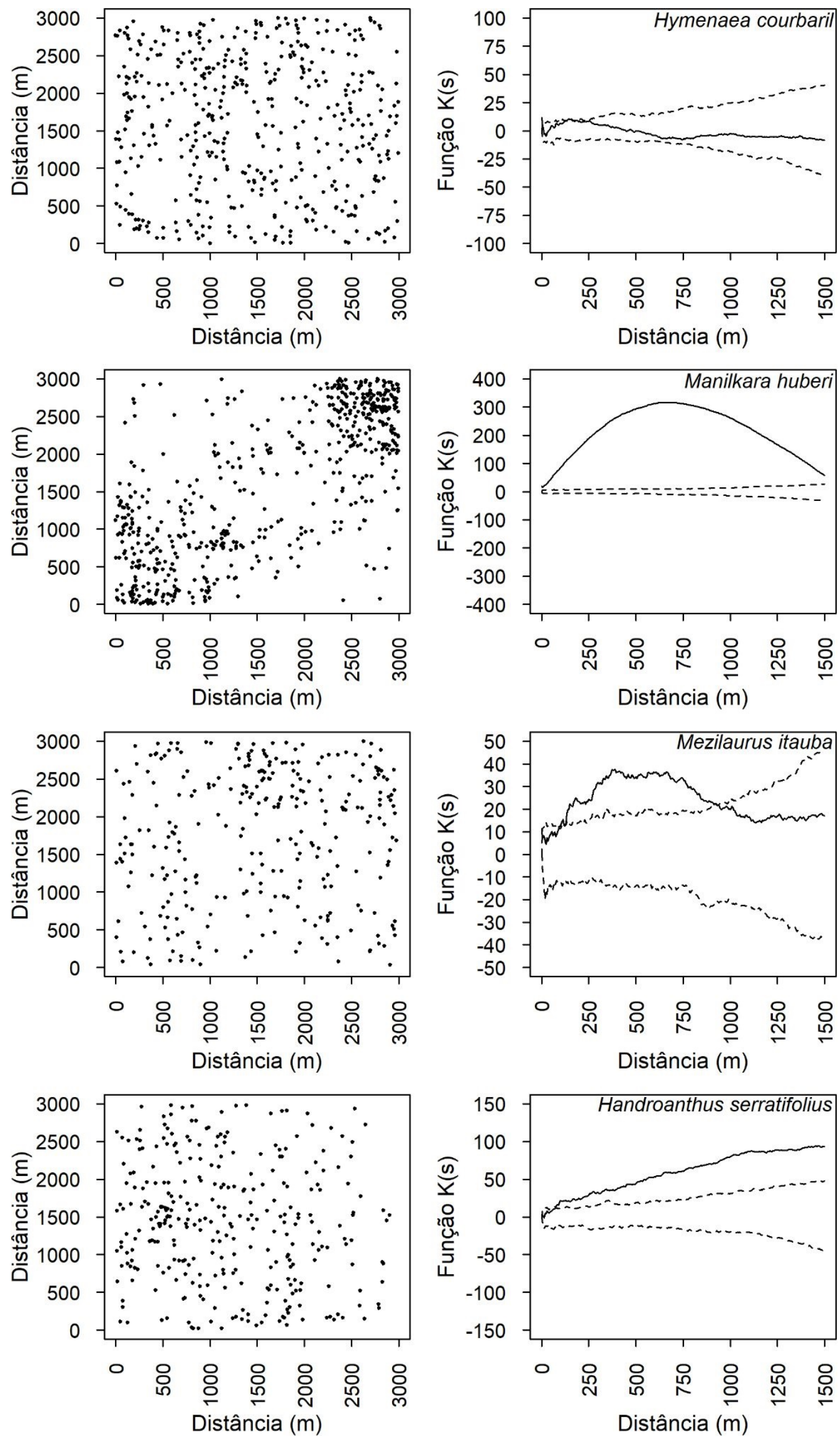

Figura 2. Função K de Ripley e padrão de pontos de todas as espécies avaliadas na Floresta Nacional do Tapajós, estado do Pará. Valores da função K (linha contínua) acima da linha tracejada superior indicam agregação, abaixo da linha inferior uniformidade e entre as linhas tracejadas total aleatoriedade. 
Avaliando a distribuição espacial de $M$. itauba em duas UPAs na Floresta Nacional do Tapajós, Rodrigues (2018) registrou padrão aleatório para distâncias até $85 \mathrm{~m}$ e agregados para distâncias de 85 a 1.500 m. A variação do padrão espacial pode sugerir a existência de gradientes ambientais e relações inter e intraespecíficas (Rodrigues, 2018), além de que o estabelecimento da espécie pode ocorrer em diferentes ambientes, o que para atividades de EIR é uma vantagem. A espécie $H$. serratifolius também atendeu parcialmente à hipótese CAE, porém houve o predomínio do padrão agregado, isto é, para distâncias superiores a $\pm 125 \mathrm{~m}$ os valores de K(s) mantiveram acima da região de CAE. A aleatoriedade nos primeiros metros de distância é provavelmente relacionada à síndrome de dispersão anemocórica, pois espécies cujo diásporos são dispersos pelo vento geralmente são distribuídas aleatoriamente, uma vez que não há deposição proposital de diásporos em determinados microssítios (Bernasol \& Lima-Ribeiro, 2010). Ao longo da Amazônia, Sontag (2017) registrou padrões agregados para quatro diferentes populações de $H$. serratifolius com DAP $\geq 45 \mathrm{~cm}$.

Acredita-se que outros fatores, provavelmente edáficos, estejam influenciando o estabelecimento das plântulas e o recrutamento de árvores de $H$. serratifolius na UPA, pois segundo Vieira \& Weber (2017) a nutrição de mudas de $H$. serratifolius é influenciada pela elevação da saturação por bases do solo. É importante lembrar, nesse caso, que normalmente os solos da Amazônia brasileira possuem baixa fertilidade e saturação por base, além de elevada acidez (Tavares et al., 2019). Ainda segundo Vieira \& Weber (2017) saturações por base de $70 \%$ são as mais recomendadas, pois aumentam a concentração de N, K, Ca, Mg e S nas mudas de $H$. serratifolius para níveis que contribuam para seu crescimento e estabelecimento. O efeito do Ca, por exemplo, se dá nos pontos de crescimento das plantas, assim o sistema radicular se desenvolve mais e as mudas se tornam mais resistentes a danos físicos e variações climáticas (Vieira \& Weber, 2017).

A espécie $M$. huberi não atendeu à hipótese CAE e apresenta padrão agregado até $1.500 \mathrm{~m}$. Os valores da função $\mathrm{K}(\mathrm{s})$ da espécie $M$. huberi foram crescentes até $\pm 700 \mathrm{~m}$, e a partir daí a curva assumiu um comportamento decrescente, indicando que o tamanho dos agrupamentos dentro da UPA varia até aproximadamente $700 \mathrm{~m}$ e que após essa escala os agrupamentos são menos nítidos. A agregação é provavelmente resultado das síndromes de dispersão zoocórica e barocórica de $M$. huberi e da ocorrência de microssítios favoráveis ao estabelecimento das árvores (Seidler \& Plotkin, 2006), uma vez que essas síndromes limitam a distribuição de propágulos, ocasionando altas taxas de germinação e recrutamento em áreas próximas às árvores de origem, enquanto em outras áreas esses processos são menores e aleatórios (Negrini et al., 2012) devido à heterogeneidade das condições edafoclimáticas, como textura, profundidade, fertilidade e disponibilidade hídrica do solo, haja vista que o estabelecimento de plantas se dá conforme as características do substrato propícias ao seu desenvolvimento.

O padrão pontual de $M$. huberi sugere que a população se encontra sob influência de fatores edáficos, visto que a maioria das árvores ocorreram preferencialmente no sudoeste (UT-01) e nordeste (UT-09) da UPA, onde, provavelmente, a fertilidade e disponibilidade hídrica do solo são mais favoráveis para o crescimento de muda de $M$. huberi, quando comparado às demais UTs (Figura 2). As árvores presentes nas UTs 01 e 09 formam grupos, isto é, aglomerados, de até $700 \mathrm{~m}$. Além do mais, constatou-se que nessas duas áreas foram registradas 18 árvores de $M$. huberi com maiores diâmetros (DAP $\geq 110 \mathrm{~cm}$ ), maiores alturas e melhores qualidades de fustes, além da alta concentração de árvores com DAP $\leq 65 \mathrm{~cm}$ em suas circunvizinhanças, o que indica que essas árvores são as mais antigas e prováveis árvores-mães. Logo, é possível que os dois grupos observados sejam formados por subgrupos de até $\pm 250 \mathrm{~m}$, que podem ser geneticamente relacionados por um ancestral comum (Azevedo et al., 2008b).

O comportamento espacial registrado para todas as espécies avaliadas pode ser usado para diversos fins, desde a seleção de métodos de amostragem à definição de operações durante a EIR (Vieira et al., 2018). A extração de madeira de espécies agregadas, por exemplo, tende a seguir sua distribuição e, portanto, os impactos de exploração não são distribuídos uniformemente (Schwartz et al., 2014). Logo, embora a EIR produza menores danos às áreas 
exploradas, em pequenas partes dessas áreas, onde a exploração madeireira é mais concentrada, o dano é maior devido à agregação da espécie. Ainda, é possível afirmar que o desconhecimento do comportamento espacial durante a exploração pode resultar em maiores clareiras, redução exagerada do número de árvores e, consequentemente, aumento da distância entre árvores individuais, alterando os processos reprodutivos, como o fluxo de pólen entre árvores, o que influenciaria a produção de sementes, taxa de germinação das espécies e estrutura diamétrica (Degen \& Roubik, 2004; Murawski \& Hamrick, 1991).

\section{CONCLUSÃO}

As espécies apresentaram distribuição diamétrica tendendo à normalidade para indivíduos com DAP $\geq 20 \mathrm{~cm}$, o que sugere problemas de regeneração ocasionados, provavelmente, pela baixa produção de sementes, condições edafoclimáticas desfavoráveis ou redução da fauna polinizadora.

A espécie H. courbaril apresentou padrão espacial completamente aleatório, enquanto as espécies $M$. huberi e $H$. serratifolius exibiram distribuição agregada. A espécie $M$. itauba apresentou padrão variando em função da distância, isto é, aleatório para distâncias inferiores a $\pm 100 \mathrm{~m}$, agregado até aproximadamente $825 \mathrm{~m}$, e aleatório até $1.500 \mathrm{~m}$. Acreditase que os padrões espaciais encontrados são resultados das síndromes de dispersão das espécies e condições edafoclimáticas da UPA.

\section{REFERÊNCIAS}

Abreu, J. C., Guedes, M. C., Guedes, A. C. L., \& Batista, E. M. (2014). Estrutura e distribuição espacial de andirobeira (Carapa spp.) em floresta de várzea do estuário amazônico. Ciência Florestal, 24(4), 1009-1019. http://dx.doi.org/10.5902/1980509816614.

Almeida, W. C., Carvalho, J. O. P., Ferreira, M. S. G., Oliveira, L. C., Vieira, I. C. G., Miranda, I. L., Soares, M. H. M., Costa, D. H. M., \& Pantoja, J. R. S. (2001). Importância ecológica e econômica e uso de espécies arbóreas existentes em uma floresta primária na área do igarapé do Mondrongo, município de Oriximiná, Pará. Belém: Embrapa Amazônia Oriental.

Alvares, C. A., Stape, J. L., Sentelhas, P. C., Gonçalves, J. L. M., \& Sparovek, G. (2013). Köppen's climate classification map for Brazil. Meteorologische Zeitschrift, 22(6), 711-728. http://dx.doi.org/10.1127/0941-2948/2013/0507.

Alves Júnior, F. T., Brandão, C. F. L. S., Rocha, K. D., Silva, J. T., Maragon, L. C., \& Ferreira, R. L. C. (2007). Estrutura diamétrica e hipsométrica do componente arbóreo de um fragmento de Mata Atlântica, Recife-PE. Cerne, 13(1), 83-95.

Angiosperm Phylogeny Group - APG IV. (2016). An update of the Angiosperm Phylogeny Group classification for the orders and families of flowering plants: APG IV. Botanical Journal of the Linnean Society, 181(1), 1-20. http://dx.doi.org/10.1111/boj.12385.

Araújo, E., David, H., Péllico, S., Morais, V., \& Scolforo, J. (2014). Padrão espacial de espécies arbóreas em fragmento de floresta estacional semidecidual. Revista de Ciências Agrárias, 57(2), 166-171. http://dx.doi.org/10.4322/rca.2014.010.

Azevedo, C. P., Sanquetta, C. R., Silva, J. N. M., \& Machado, S. A. (2008a). Efeito da exploração de madeira e dos tratamentos silviculturais no agrupamento ecológico de espécies. Floresta, 38(1), 1-17. http://dx.doi.org/10.5380/rf.v38i1.11027.

Azevedo, V. C. R., Kanashiro, M., Grattapaglia, D., \& Ciampi, A. Y. (2008b). Variabilidade no cpDNA em Manilkara huberi, espécie sob manejo sustentável na Amazônia brasileira. Pesquisa Agropecuária Brasileira, 43(7), 859-867. http://dx.doi.org/10.1590/S0100-204X2008000700010.

Bernasol, W. P., \& Lima-Ribeiro, M. S. (2010). Spatial distribution pattern and size structure of tree species and its structuring factors in a fragment of the Brazilian cerrado. Hoehnea, 37(2), 181-198. http://dx.doi.org/10.1590/S2236-89062010000200001.

Brasil. (2006, 13 de dezembro). Instrução normativa n 5, de 11 de dezembro de 2006. Altera as Diretrizes para o Manejo Florestal em escala Empresarial na Amazônia. Diário Oficial [da] República Federativa do Brasil (No. 238, pp. 155-158), Brasília, seção 1. 
Braz, E. M., Canetti, A., Mattos, P. P., Basso, R. O., \& Figueiredo Filho, A. (2018). Alternative criteria to achieve sustainable management of Mezilaurus itauba in the Brazilian Amazon. Pesquisa Florestal Brasileira, 38(1), 1-8. http://dx.doi.org/10.4336/2018.pfb.38e201801648.

Carvalho, F. A. (2010). Síndrome de dispersão de espécies arbóreas de florestas ombrófilas submontanas do estado do Rio de Janeiro. Revista Árvore, 34(6), 1017-1023. http://dx.doi.org/10.1590/S0100-67622010000600007.

Costa, S. N., Rabelo, F. G., Lima, R. B., Silva, D. A. S., Lima, C. W. P., \& Santos, S. M. (2019). Estrutura populacional de Manilkara huberi (Ducke) A. Chev. E Dinizia excelsa Ducke em floresta de terra firme no Amapá. Nativa (Sinop), 7(4), 445-451. http://dx.doi.org/10.31413/nativa.v7i4.7638.

Cunha, U. S., Machado, S. A., Figueiredo Filho, A. F., \& Freitas, J. V. (2012). Diameter structure and phytosociological frequency of a "terra firme" amazon forest, before and after selective cutting. Floresta, 42(2), 293-304. http://dx.doi.org/10.5380/rf.v42i2.21463.

Cysneiros, V. C., Amorim, T. A., Mendonça Júnior, J. O., Guai, T. D., Moraes, J. C. R., Braz, D. M., \& Machado, S. M. (2017). Distribuição diamétrica de espécies da Floresta Ombrófila Densa no Sul do Estado do Rio de Janeiro. Pesquisa Florestal Brasileira, 37(89), 1-10. http://dx.doi.org/10.4336/2017.pfb.37.89.1070.

Dalla Lana, M., Brandão, C. F. L. S., Péllico Netto, S., Marangon, L. C., \& Souza Retslaff, F. A. (2013). Distribuição diamétrica de Escheweilera ovata em um fragmento de Floresta Ombrófila Densa Igarassu, PE. Floresta, 43(1), 59-68. http://dx.doi.org/10.5380/rf.v43i1.25252.

Dantas, A. R., Marangon, L. C., Guedes, M. C., Feliciano, A. L. P., \& Lira-Guedes, A. C. (2017). Spatial distribution of a population of Pentaclethra macroloba (Willd.) Kuntze in a floodplain forest of the Amazon Estuary. Revista Árvore, 41(4), 1-11.

Degen, B., \& Roubik, D. W. (2004). Effects of animal pollination on pollen dispersal, selfing, and effective population size of tropical trees: a simulation study. Biotropica, 36(2), 165-179. http://dx.doi.org/10.1111/j.1744-7429.2004.tb00309.x.

Ebert, A., Brito da Costa, R., \& Brondani, G. (2016). Spatial distribution pattern of Mezilaurus itauba (Meins.) Taub. Ex Mez. in a seasonal forest area of the southern Amazon, Brazil. iForest Biogeosciences and Forestry, 9(3), 497-502. http://dx.doi.org/10.3832/ifor1427-008.

Ferraz, I. D. K., Camargo, J. L. C., \& Sampaio, P. T. B. (2002). Sementes e plântulas de andiroba (Carapa guianensis Aubl. e Capara procera D. C.): aspectos botânicos, ecológicos e tecnológicos. Acta Amazonica, 32(4), 647-661.

Gül, A. U., Misir, M., Misir, N., \& Yavuz, H. (2005). Calculation of uneven-aged stand structures with the negative exponential diameter distribution and Sterba's modified competition density rule. Forest Ecology and Management, 214(1), 212-220. http://dx.doi.org/10.1016/j.foreco.2005.04.012.

Hirai, E. H., Carvalho, J. O. P., \& Pinheiro, K. A. O. (2008). Estrutura da população de maçaranduba (Manilkara huberi Standley) em 84 ha de floresta natural na Fazenda Rio Capim, Paragominas, PA. Revista de Ciências Agrárias Amazonian Journal of Agricultural and Environmental Sciences, 49(1), 65-76.

International Union for Conservation of Nature - IUCN. (2018). Red List of threatned species. Retrieved in 2019, December 30, from http//www.redlist.org/

Jorge, L. A. B., Millani, T. M., Fonseca, R. C. B., \& Arruda, A. A. (2015). Estrutura diamétrica e arranjo espacial das espécies mais abundantes de um fragmento de Floresta Estacional Semidecidual em Botucatu, SP. Floresta e Ambiente, 22(3), 355-367. http://dx.doi.org/10.1590/2179-8087.027713.

Lan, G., Getzin, S., Wiegand, T., Hu, Y., Xie, G., Zhu, H., \& Cao, M. (2012). Spatial distribution and interspecific associations of tree species in a Tropical Seasonal Rain Forest of China. PLoS One, 7(9), e46074. PMid:23029394. http://dx.doi.org/10.1371/journal.pone.0046074.

Lima-Ribeiro, M. S., Rezende, S. L., \& Bernasol, W. P. (2011). Estrutura espacial e deposição de sementes de Hymenaea courbaril L. em um fragmento florestal no sudoeste goiano. Acta Scientiarum. Biological Sciences, 33(1), 41-47. http://dx.doi.org/10.4025/actascibiolsci.v33i1.5881.

Martins, S. S., Couto, L., Machado, C. C., \& Souza, A. L. (2003). Efeito da exploração floresta seletiva em uma floresta estacional semidecidual. Revista Árvore, 27(1), 65-70. http://dx.doi.org/10.1590/S010067622003000100009.

Moraes, G., Schorr, L., Aguiar, J., Cuchi, T., \& Melo, L. (2017). Mudanças na estrutura diamétrica e no arranjo espacial de Manilkara elata em área manejada na Floresta Nacional do Tapajós. Enciclopédia Biosfera, 14(25), 950-959. http://dx.doi.org/10.18677/EnciBio_2017A76.

Murawski, D. A., \& Hamrick, J. L. (1991). The effect of the density of flowering individuals on the mating systems of nine tropical tree species. Heredity, 67(2), 167-174. http://dx.doi.org/10.1038/hdy.1991.76. 
Negrini, M., Aguiar, M. D., Vieira, C. T., Silva, A. C., \& Higuchi, P. (2012). Dispersão, distribuição espacial e estratificação vertical da comunidade arbórea em um fragmento florestal no Planalto Catarinense. Revista Árvore, 36(5), 919-930. http://dx.doi.org/10.1590/S0100-67622012000500014.

Oksanen, J., Blanchet, F. G., Kindt, R., Legendre, P., Minchin, P. R., O'Hara, R. B., Simpson, G. L., Solymos, P., Stevens, M. H. H., \& Wagner, H. (2018). vegan: Community Ecology Package. Vienna: R Foundation for Statistical Computing. Retrieved in 2018, November 10, from https://CRAN.Rproject.org/package=vegan

Oliveira, W. L., Medeiros, M. B., Moser, P., Pinheiro, R., \& Olsen, L. B. (2011). Regeneração e estrutura populacional de jatobá-da-mata (Hymenaea courbaril L.), em dois fragmentos com diferentes graus de perturbação antrópica. Acta Botanica Brasílica, 25(4), 876-884. http://dx.doi.org/10.1590/S010233062011000400014.

Orellana, E., Figueiredo Filho, A., Netto, S. P., \& Dias, A. N. (2014). Modelagem da distribuição diamétrica de espécies florestais em um fragmento de floresta ombrófila mista. Revista Árvore, 38(2), 297-308. http://dx.doi.org/10.1590/S0100-67622014000200010.

Pinheiro, K. A. O., Carvalho, J. O. P., Quanz, B., Francez, L. M. B., \& Schwartz, G. (2007). Fitossociologia de uma área de preservação permanente no leste da Amazônia: indicação de espécies para recuperação de áreas alteradas. Floresta, 37(2), 175-187. http://dx.doi.org/10.5380/rf.v37i2.8648.

Pinto, A. A., Teles, B. R., Anjos, N., \& Couceiro, S. R. M. (2013). Predação de sementes de andiroba [Carapa guianensis Aubl. e Carapa procera DC. (Meliaceae)] por insetos na Amazônias. Revista Árvore, 37(6), 1115-1123. http://dx.doi.org/10.1590/S0100-67622013000600013.

R Core Team. (2018). R: a language and environment for statistical computing. Vienna: R Foundation for Statistical Computing. Retrieved in 2018, January 1, from https://www.R-project.org/

Ripley, B. D. (1977). Modelling spatial patterns. Journal of the Royal Statistical Society. Series B. Methodological, 39(2), 172-212. http://dx.doi.org/10.1111/j.2517-6161.1977.tb01615.x.

Ripley, B. D. (1979). Tests of 'Randomness' for Spatial Point Patterns. Journal of the Royal Statistical Society. Series B. Methodological, 41(3), 368-374. http://dx.doi.org/10.1111/j.25176161.1979.tb01091.x.

Rodrigues, B. L. (2018). Padrões espaciais e quantificação de árvores comerciais na Amazônia (Dissertação de mestrado). Universidade Federal dos Vales do Jequitinhonha e Mucuri, Diamantina.

Rowlingson, B., \& Diggle, P. (2018). splancs: spatial and space-time point pattern analysis. Vienna: R Foundation for Statistical Computing. Retrieved in 2018, November 10, from https://CRAN.Rproject.org/package=splancs

Santos, L. S., Martorano, L. G., Gutierrez, C. B. B., Pontes, A. N., Silva, O. M., \& Gutierrez, D. M. G. (2017). Aspectos fisiográficos da Floresta Nacional do Tapajós e seu entorno - Oeste do Pará, Brazil. Revista Espacios, 38(2), 26.

Schwartz, G., Lopes, J. C., Kanashiro, M., Mohren, G. M., \& Peña-Claros, M. (2014). Disturbance level determines the regeneration of commercial tree species in the Eastern Amazon. Biotropica, 46(2), 148-156. http://dx.doi.org/10.1111/btp.12096.

Schwartz, G., Nascimento, N. A., \& Menezes, A. J. E. A. (2008). Estrutura populacional de espécies de interesse florestal não madeireiro no sudoeste do Pará, Brasil. Amazônia: Ciência \& Desenvolvimento, 4(7), 117-130.

Seidler, T. G., \& Plotkin, J. B. (2006). Seed dispersal and spatial pattern in tropical trees. PLoS Biology, 4(11), e344. PMid:17048988. http://dx.doi.org/10.1371/journal.pbio.0040344.

Sontag, V. E. (2017). Alterações na legislação brasileira de manejo florestal e seus efeitos na distribuição espacial e polinização de espécies madeireiras amazônicas (Dissertação de mestrado). Escola Superior de Agricultura de "Luiz de Queiroz", Universidade de São Paulo, Piracicaba.

Souza, A. L., Medeiros, R. M., Matos, L. M. S., Silva, K. R., Corrêa, P. A., \& Faria, F. N. (2014). Estratificação volumétrica por classes de estoque em uma Floresta Ombrófila Densa, no Município de Almeirim, Estado do Pará, Brasil. Revista Árvore, 38(3), 533-541. http://dx.doi.org/10.1590/S010067622014000300016.

Souza, D. R., \& Souza, A. L. (2004). Estratificação vertical em floresta ombrófila densa de terra firme não explorada, Amazônia Oriental. Revista Árvore, 28(5), 691-698. http://dx.doi.org/10.1590/S010067622004000500008.

Souza, D. R., Souza, A. L., Gama, J. R. V., \& Leite, H. G. (2003). Emprego de análise multivariada para estratificação vertical de florestas ineqüiâneas. Revista Árvore, 27(1), 59-63. http://dx.doi.org/10.1590/S0100-67622003000100008. 
Stamatellos, G., \& Panourgias, G. (2005). Simulating spatial distributions of forest trees by using data from fixed area plots. Forestry, 78(3), 305-312. http://dx.doi.org/10.1093/forestry/cpi028.

Tavares, L. D. S., Weber, O. L. S., Valadão, F. C. A., \& Espinosa, M. M. (2019). Análise multivariada de espécies florestais nativas em relação aos atributos químicos e texturais do solo na região de Cotriguaçi - MT. Ciência Florestal, 29(1), 281-291. http://dx.doi.org/10.5902/198050983577.

Téo, S. J., Marcon, F., Schneider, C. R., Santos, F. B., Chiarello, K. M. A., \& Fiorentin, L. D. (2014). Modelagem da distribuição diamétrica de um fragmento de Floresta Ombrófila Mista em Lebon Régis, SC. Floresta, 45(2), 337-348. http://dx.doi.org/10.5380/rf.v45i2.34733.

Vieira, C., \& Weber, O. (2017). Saturação por bases no crescimento e na nutrição de mudas de ipêamarelo. Floresta e Ambiente, 24(1), 1-10.

Vieira, D. S. V., Oliveira, M. L. R., Gama, J. R. V., Machado, E. L. M., \& Gorgens, E. B. (2017). Patrón espacial y métodos de muestreo para Bertholletia excelsa (castaña de Pará) en la Mesorregión del Bajo Amazonas, estado de Pará, Brasil. Bosque, 38(1), 97-107. http://dx.doi.org/10.4067/S071792002017000100011.

Vieira, D. S., Oliveira, M. L. R., Gama, J. R. V., Oliveira, B. O., Rego, A. K. C., \& Bezerra, T. G. (2018). Sampling processes for Carapa guianensis Aubl. in the Amazon. Cerne, 24(3), 169-179. http://dx.doi.org/10.1590/01047760201824032514.

Wendling, W. T., Emerenciano, D. B., \& Hosokawa, R. T. (2011). Ajuste da função de distribuição diamétrica Weibull por planilha eletrônica. Floresta, 41(2), 205-220. http://dx.doi.org/10.5380/rf.v41i2.21869.

Zhang, Z., Hu, G., Zhu, J., \& Ni, J. (2013). Aggregated spatial distributions of species in a subtropical karst forest, southwestern China. Journal of Plant Ecology, 6(2), 131-140. http://dx.doi.org/10.1093/jpe/rts027.

Contribuição dos Autores: DSV: conceituação, curadoria de dados, análise formal, metodologia, supervisão, escrita - primeira redação, escrita revisão e edição; KMAG e LES: conceituação, metodologia, supervisão e escrita primeira redação; MLRO, JRVG, ELMM e BOL: supervisão, escrita - primeira redação, escrita - revisão e edição; CCM: curadoria de dados, metodologia e escrita - primeira redação; e AESF: supervisão e escrita - primeira redação. 